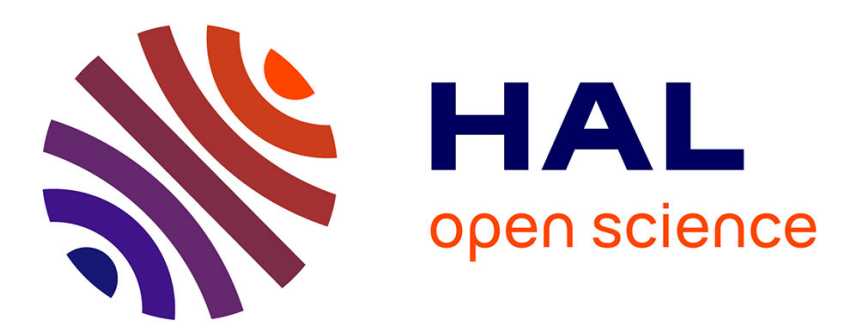

\title{
A condensed transfer function method as a tool for solving vibroacoustic problems
}

Valentin Meyer, Laurent Maxit, J.-L. Guyader, T. Leissing, C. Audoly

\section{To cite this version:}

Valentin Meyer, Laurent Maxit, J.-L. Guyader, T. Leissing, C. Audoly. A condensed transfer function method as a tool for solving vibroacoustic problems. Proceedings of the Institution of Mechanical Engineers, Part C: Journal of Mechanical Engineering Science, 2015, 10.1177/0954406215615627. hal-01277091

\section{HAL Id: hal-01277091 \\ https://hal.science/hal-01277091}

Submitted on 22 Feb 2016

HAL is a multi-disciplinary open access archive for the deposit and dissemination of scientific research documents, whether they are published or not. The documents may come from teaching and research institutions in France or abroad, or from public or private research centers.
L'archive ouverte pluridisciplinaire HAL, est destinée au dépôt et à la diffusion de documents scientifiques de niveau recherche, publiés ou non, émanant des établissements d'enseignement et de recherche français ou étrangers, des laboratoires publics ou privés. 


\title{
A Condensed Transfer Function Method as a Tool for Solving Vibroacoustic Problems
}

\author{
Valentin Meyer $^{a}$, Laurent Maxit, Jean-Louis Guyader \\ INSA Lyon, Laboratoire Vibrations Acoustique, 25 bis, av. Jean Capelle, 69621 Villeurbanne, France \\ Thomas Leissing, Christian Audoly \\ DCNS Research, France \\ a) Author to whom correspondance should be addressed. Electronic mail: valentin.meyer@insa-lyon.fr
}

\begin{abstract}
Substructuring approaches are nowadays widely used to predict numerically the vibroacoustic behavior of complex mechanical systems. Some of these methods are based on admittance or mobility frequency transfer functions at the coupling interfaces. They have already been used intensively to couple subsystems linked by point contacts and enable to solve problems at higher frequency while saving computation costs. In the case of subsystems coupled along lines, a Condensed Transfer Function (CTF) method is developed in the present paper. The admittances on the coupling line are condensed in order to reduce the number of coupling forces evaluated. Three variants are presented, where the transfer functions are condensed using three different functions. After describing the principle of the CTF method, simple structures will be given as test cases for validation.
\end{abstract}

\section{Keywords}

Vibroacoustic, Sub-structuring, Admittance, Numerical Analysis 


\section{Introduction}

Sub-structuring methods in dynamics or vibroacoustics have been intensively studied over the last decades in order to push always further the computational limitations of numerical models. The most widespread techniques today are certainly the modal synthesis methods. They are based on the Rayleigh-Ritz method of modal estimation and consist in splitting the system and calculating modal basis with different boundary conditions. Hurty 11 was one of the first to introduce the Component Mode Synthesis (CMS) and was then followed by many developments. Craig and Bampton ${ }^{2}$ formulated a variant of the method using the constraint modes and the shape modes with fixed interfaces. Gladwell ${ }^{3}$ developed the branch mode analysis, which is particularly adapted to systems defined by a main component on which secondary components are mounted. The basis for the main component is characterized by its modes with free interfaces while the secondary components are characterized by the constraint modes and the shape modes with fixed interface (similarly to Craig and Bampton). MacNeal ${ }^{4}$ presented a hybrid method, mixing free and fixed interfaces with several corrections, to minimize the effects of modal truncature and improve the convergence. More recently, Tournour et al $!^{[5}$ gave convergence criteria and benchmark data for the free CMS, which main advantage lies in the fact that the degrees of freedom at the interface are eliminated from the final system of equations during the assembly process.

Modal basis can however not always be calculated easily and methods based on impedance or mobility concepts are then a common alternative to the CMS techniques. ${ }^{6 / 7}$ They offer the possibility to couple subsystems which characteristics are determined by different means (numerical, analytical, experimental). Petersson and Plunt ${ }^{8 / 9}$ characterize the vibratory power input in a structure by defining effective mobilities to account for multi-point coupled structure-borne sound sources. The concepts have been experimentally investigated and show a great practical interest. For linear problems, Ouisse et al ${ }^{10}$ developed the Patch Transfer Functions (PTF) method based on the mobility method to couple subsystems along surfaces. These surfaces, that can be either the junction between a structure and a fluid domain or between two fluid domains, are divided into patches to calculate transfer functions. The method is first validated on an acoustic cavity divided in two domains, before being applied to the automotive industry. Chazot and Guyader ${ }^{11}$ later reused the method in order to evaluate the transmission loss through double panels. Aucejo et al ${ }^{12}$ focused on the heavy fluid loading and improved the convergence of the method by introducing residual mode shapes. Maxit et al ${ }^{13}$ have shown that the size of the patches can be increased by choosing coupling surfaces outside the acoustic near-field. An example of application is given on the sound transmission in the ballasts of a submarine vehicle. The results of these studies point out a convergence criterion on the size of the patches, namely that they should remain smaller than half a wavelength. More recently, the PTF approach has been applied to solve a vibroacoustic problem involving porous materials, $\frac{14}{14}$ or has been used to allow coupling between geometrical acoustics and another domain.15

In this paper, a sub-structuring approach called the Condensed Transfer Function 
(CTF) method is proposed as an extension to couple structures along a line. This development is motivated by the fact that line coupling can often be met in industrial applications. In the aeronautical and naval industry for instance, some internal frames that stiffen the structure and support the machinery are connected to the shell along lines. A set of orthonormal functions called condensation functions, that depend on the curvilinear abscissa along the coupling line, is considered. This set is used as a basis for approximating and decomposing the displacements and the applied forces at the line junctions. Thanks to the definition and calculation of condensed transfer functions for each uncoupled subsystem and by using the superposition principle for passive linear system, the behavior of the coupled subsystems can be deduced. Three examples of condensation sets are given and the method is applied to a plate in order to study the convergence with respect to the number of transfer functions considered. Part of this work has been presented previously at NOVEM2015 16

\section{Principle of the Condensed Transfer Function (CTF) method}

\subsection{Classical admittance approach for point coupled subsystems}

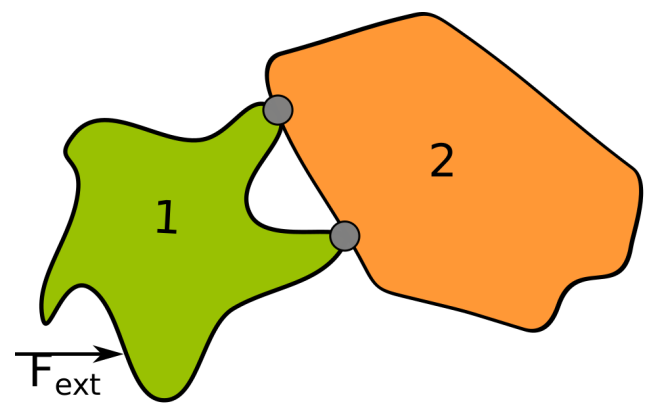

Figure 1: Subsystems linked by two point contacts.

Introduced first by Firestone $\frac{17}{17}$ the admittance method has been used to calculate the harmonic response of subsystems coupled by contact points (see Figures 1 and 2), and under an external load $F_{\text {ext }}$. The contacts are assumed to be perfect, without friction loss, and bilateral (meaning that the forces transmitted have the same amplitude but are of opposite sign).

For general 3-D problems, $\mathbf{U}_{n}^{\alpha}=\left[U_{n, p}^{\alpha}\right]_{p}$ is the vector of the 3 translations and 3 rotations of space at the point $n$ and $\mathbf{F}_{m}^{\alpha}=\left[F_{m, q}^{\alpha}\right]_{q}$ the vector of the 3 forces and 3 moments applied on $m$. The admittance $\mathbf{Y}_{n m}^{\alpha}$ of a subsystem $\alpha$ is defined as the ratio of the displacement at the point $n$ over the force applied at the point $m$ : 


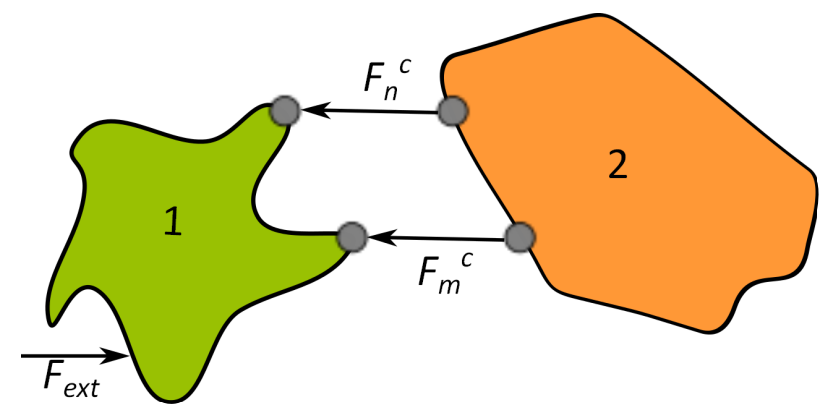

Figure 2: Coupling forces between the two subsystems.

$$
\mathbf{Y}_{n m}^{\alpha}=\left[\frac{U_{n, p}^{\alpha}}{F_{m, q}^{\alpha}}\right]_{1 \leq p \leq 6,1 \leq q \leq 6} .
$$

For two coupling points $(n, m)$, the admittance $\mathbf{Y}_{n m}^{\alpha}$ is thus a $6 \times 6$ matrix to account for all the degrees of freedom.

Moreover, the displacement vector at point $n$ of the uncoupled subsystems when only external loading is applied, is called free displacement and is written $\tilde{\mathbf{U}}_{n}^{\alpha}$. Based on the superposition principle, the displacements of an uncoupled subsystem $\alpha$ at the contact point $n$ can be written as

$$
\mathbf{U}_{n}^{\alpha}=\tilde{\mathbf{U}}_{n}^{\alpha}+\mathbf{Y}_{n n}^{\alpha} \mathbf{F}_{n}^{\alpha}+\mathbf{Y}_{n m}^{\alpha} \mathbf{F}_{m}^{\alpha}, \alpha \in\{1,2\} .
$$

The displacements at the contact point $m$ are simply derived from the previous equation by interchanging $n$ and $m$.

The displacement continuity and force equilibrium between subsystem 1 and 2 are written for all connection points as

$$
\left\{\begin{array}{c}
\mathbf{U}_{n}^{1}=\mathbf{U}_{n}^{2} \\
\mathbf{F}_{n}^{1}=-\mathbf{F}_{n}^{2}=\mathbf{F}_{n}^{c}
\end{array}, \forall n .\right.
$$

Equations 2 and 3 yield to Equation 4 and the coupling forces $\mathbf{F}_{n}^{c}$ at the connection points can thus be deduced by inverting the admittance term in the following equation:

$$
\left(\mathbf{Y}^{1}+\mathbf{Y}^{2}\right) \mathbf{F}^{c}=\tilde{\mathbf{U}}^{2}-\tilde{\mathbf{U}}^{1}
$$

where the notation without subscripts denotes vector and matrices assembled for all connection points. In this case, as no external load is applied on subsystem $2, \tilde{\mathbf{U}}^{2}=0$.

This method can easily be extended to more than two coupling points by increasing the size of the admittance matrices and free displacement vectors 18 


\subsection{Extension to line coupled subsystems}

In industrial applications, it is likely that subsystems are coupled along lines rather than on points, as shown on Figures 3 and 4. That is why the admittance method needs to be extended to allow line coupling. A set of $N$ functions called condensation functions is considered: $\left\{\varphi_{n}\right\}_{1 \leq n \leq N}$. They are functions of the curvilinear abscissa $s$ along the coupling line $\Gamma$. The number of transfer functions $N$ plays a key role in the convergence of the method and its value will be discussed later in this paper. The set of condensation functions is supposed to be orthonormal. For an uncoupled subsystem $\alpha$, a condensed transfer function is defined as follows:

$$
\mathbf{Y}_{n m}^{\alpha}=\left[\frac{\left\langle\bar{U}_{m, q, p}^{\alpha}, \varphi_{n}\right\rangle}{\left\langle F_{m, q}^{\alpha}, \varphi_{m}\right\rangle}\right]_{1 \leq p \leq 6,1 \leq q \leq 6}=\left[\left\langle\bar{U}_{m, q, p}^{\alpha}, \varphi_{n}\right\rangle\right]_{p, q},
$$

where $\langle\bullet, \bullet\rangle$ is a scalar product. $\mathbf{F}_{m}^{\alpha}$ is the vector of the efforts on $\Gamma$, where the components in each direction of space is equal to $\varphi_{m}$. The indexes $p$ and $q$ denote directions of space. $\overline{\mathbf{U}}_{m, q}^{\alpha}$ is the displacements vector of the junction $\Gamma$ when the subsystem is excited in the direction $q$ by a force of magnitude $F_{m, q}^{\alpha}$.

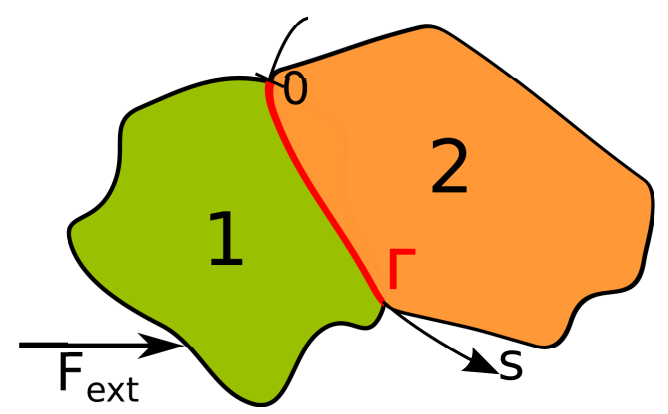

Figure 3: Subsystems coupled along a line $\Gamma$.
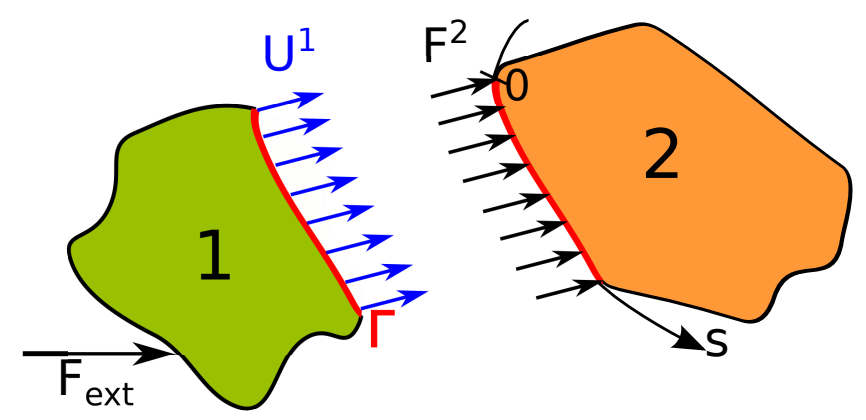

Figure 4: Uncoupled systems, interface forces and displacements at the junction. 
It is assumed that the displacements vector $\mathbf{U}^{\alpha}$ at the junction can be written for each subsystem $\alpha \in\{1,2\}$ as a linear combination of the condensation functions:

$$
\left\{\begin{array}{l}
\mathbf{U}^{1}(s)=\sum_{n=1}^{N} \mathbf{u}_{n}^{1} \varphi_{n}(s) \\
\mathbf{U}^{2}(s)=\sum_{n=1}^{N} \mathbf{u}_{n}^{2} \varphi_{n}(s)
\end{array}\right.
$$

where $s$ is the coordinate along the arc $\Gamma$, and $\mathbf{u}_{n}^{\alpha}$ the condensed displacements vector of subsystem $\alpha$ associated to the condensation function $\varphi_{n}$.

Let us write $\mathbf{F}^{\alpha}(s)=\sum_{n=1}^{N} \mathbf{f}_{n}^{\alpha} \varphi_{n}(s)$ the efforts applied on the junction of the subsystem $\alpha$. In response to these linear excitation forces, the superposition principle for passive linear systems enables to write the condensed displacements $\mathbf{u}_{n}^{\alpha}$ as

$$
\left\{\begin{array}{l}
\mathbf{u}_{n}^{1}=\tilde{\mathbf{u}}_{n}^{1}+\sum_{m=1}^{N} \mathbf{Y}_{n m}^{1} \mathbf{f}_{m}^{1} \\
\mathbf{u}_{n}^{2}=\tilde{\mathbf{u}}_{n}^{2}+\sum_{m=1}^{N} \mathbf{Y}_{n m}^{2} \mathbf{f}_{m}^{2}
\end{array} \quad, \forall s \in \Gamma, \forall n \in \llbracket 1 ; N \rrbracket,\right.
$$

where $\tilde{\mathbf{u}}_{n}^{\alpha}=\left[\left\langle\tilde{U}_{p}^{1}, \varphi_{n}\right\rangle\right]_{p}$ are the free displacements of the uncoupled subsystems. As on this example the subsystem 2 has no external load: $\tilde{\mathbf{u}}_{n}^{2}=0, \forall n$.

Besides, the displacement continuity and force equilibrium lead to

$$
\left\{\begin{array}{c}
\mathbf{U}^{1}(s)=\mathbf{U}^{2}(s) \\
\mathbf{F}^{1}(s)+\mathbf{F}^{2}(s)=0
\end{array}, \forall s \in \Gamma\right.
$$

The set of condensation functions being orthonormal, Equation 8 yields to

$$
\left\{\begin{array}{c}
\mathbf{u}_{n}^{1}=\mathbf{u}_{n}^{2} \\
\mathbf{f}_{n}^{1}=-\mathbf{f}_{n}^{2}=\mathbf{f}_{n}^{c}
\end{array} \quad, \forall n \in \llbracket 1 ; N \rrbracket\right.
$$

Assembling the vectors and matrices for all the condensation functions and injecting Equation 17 in the previous equation results in a similar formula than the classical admittance method to deduce the coupling forces between the subsystems:

$$
\left(\mathbf{Y}^{1}+\mathbf{Y}^{2}\right) \mathbf{F}^{c}=-\tilde{\mathbf{U}}^{1}
$$

To clarify this point, the general expression for the admittance matrix and the free displacement vectors are given in the following equations:

$$
\mathbf{Y}^{\alpha}=\left[\begin{array}{ccccc} 
& \vdots & \vdots & \vdots & \\
\ldots & \mathbf{Y}_{n n}^{\alpha} & \ldots & \mathbf{Y}_{n m}^{\alpha} & \ldots \\
& \vdots & \vdots & \vdots & \\
\ldots & \mathbf{Y}_{m n}^{\alpha} & \ldots & \mathbf{Y}_{m m}^{\alpha} & \ldots \\
& \vdots & \vdots & \vdots &
\end{array}\right]
$$

and 


$$
\tilde{\mathbf{U}}^{\alpha}=\left[\begin{array}{c}
\vdots \\
\tilde{\mathbf{u}}_{n}^{\alpha} \\
\vdots \\
\tilde{\mathbf{u}}_{m}^{\alpha} \\
\vdots
\end{array}\right]
$$

where it is reminded that one element of the matrix $\mathbf{Y}^{\alpha}$ is a square matrix which size depends on the number of degrees of freedom (generally 6 in 3-D problems), to take into account all the possible directions of excitations and displacements. Similarly, one element of $\tilde{\mathbf{U}}^{\alpha}$ is a 6 elements vector (still considering having a general 3-D problem) to calculate the response of the junction in all directions of space to the external load. The size of this system is therefore equal to the number of transfer functions multiplied by the number of degrees of freedom.

In practice, the admittance matrices are calculated by exciting each uncoupled subsystems by all the condensation functions one after the other. Indeed, as the set is orthonormal, the denominator of the admittance term is in this case equal to 1 . The admittance is deduced by projecting the displacements on the condensation functions.

Finally the behavior of subsystem 1 is obtained by applying the external loading and the coupling forces thus calculated. Similarly, the behavior of subsystem 2 is known by applying the coupling forces at the junction, and the whole system has thus been described.

The method has been presented in the case of the coupling between only two subsystems but can easily be extended to more complex systems, with more than one joint. Examples of condensation functions with their associated scalar product are given in the next section, before being applied to a plane plate test case.

\section{$3 \quad$ Numerical study on a plate}

\subsection{Test case parameters}

To illustrate the method presented above, let us consider in this section two rectangular plates made of the same material and thickness and which characteristics and dimensions are given in Table 1. The plates lie in the $z=0$ plane and the boundary conditions are free on all edges. The aim is to couple the two plates together along their longest edge, as shown on Figure 6.

A reference calculation is made by the Finite Element Method (FEM) on a $1.5 \times 2.5$ $\mathrm{m}^{2}$ plate. Harmonic responses are calculated for frequencies lying between $10 \mathrm{~Hz}$ and 1500 $\mathrm{Hz}$, with almost 400 values logarithmically spread over the domain. This choice ensures enough values to describe properly the resonances of the system regarding the value of the structural damping coefficient,$\frac{19}{}$ which is accounted for as a complex factor in the 
Table 1: Material characteristics and plates dimensions.

\begin{tabular}{cccc}
\hline Parameter & Notation & Value & Unit \\
\hline Young modulus & $E$ & $2.1 \times 10^{11}$ & $\mathrm{~Pa}$ \\
Poisson coefficient & $\nu$ & 0.3 & - \\
Density & $\rho_{p}$ & 7800 & $\mathrm{~kg} / \mathrm{m}^{3}$ \\
Structural damping coeff. & $\eta$ & 0.02 & - \\
Length of plate 1 \& 2 & $L$ & 1.5 & $\mathrm{~m}$ \\
Width of plate 1 & $l_{1}$ & 1.2 & $\mathrm{~m}$ \\
Width of plate 2 & $l_{2}$ & 1.3 & $\mathrm{~m}$ \\
Thickness & $h$ & 0.017 & $\mathrm{~m}$ \\
\hline
\end{tabular}

stiffness matrix. One layer of quadrilateral isotropic shell elements of thickness $h$ is chosen with a linear interpolation function. The Mindlin-Timoshenko formulation ${ }^{20}$ is considered to take into account the effects of shear and rotational inertia. A virtual stiffness, which has no influence on the flexural waves, is added on the normal rotation in order to avoid numerical problems. The mesh is fine enough in order to respect the criterion of 6 elements per bending wavelength which is commonly used for such problems.

The two subsystems are modeled by FEM and the condensed admittances and free displacements need to be calculated for each of the two uncoupled subsystems. Thanks to its good convergence and calculation time, the frequency responses of the subsystems under different excitations are estimated by modal superposition including quasi-static modes. ${ }^{21}$ This method consists in adding very low frequency mode shapes to the modal basis in order to compensate for the basis truncation. Results of the CTF method are presented in the next subsection. The basis truncation is made so that the frequency of the highest mode is at least 1.5 times higher than the highest frequency. In the case of the two plates described previously, the basis for plate 1 counts 116 modes (highest eigenfrequency at $f=2848.6$ $\mathrm{Hz}$ ) and 125 modes for plate 2 (highest eigenfrequency at $f=2839.3 \mathrm{~Hz}$ ). Three examples of condensation functions are given in the next subsection and the results for the present test case are discussed.

\subsection{Three examples of condensation functions}

\subsubsection{Gate functions:}

The gate functions are defined depending on their length, as follows:

$$
\varphi_{n}(s)= \begin{cases}\frac{1}{\sqrt{L_{s}}} & \text { if }(n-1) L_{s} \leq s<n L_{s} \\ 0 & \text { elsewhere }\end{cases}
$$

The scalar product associated to the gate function is defined in Equation 14. Considering the set of piecewise continuous functions on segments $[a, b[$, it can be easily verified that the gate functions form an orthonormal set for this scalar product. 


$$
\langle f, g\rangle=\int_{\Gamma} f(s) g^{*}(s) d s
$$

where ${ }^{*}$ means the complex conjugate.

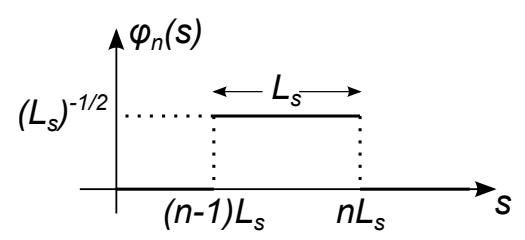

Figure 5: Gate function of length $L_{s}$.

The shape of one of these functions can be seen on the plot on Figure 5. Building the admittance matrix of each subsystem in this case can be seen as dividing the coupling line into $N$ segments, as shown on Figure 6. The segments are excited one after the other, and the observation of the displacements allows to obtain a $6 N \times 6 N$ matrix, considering a 6 degrees of freedom problem. Each term of the free displacement vector is the result of the integral of the displacement on one segment due to the external load (point force at the point $\mathrm{A}$ in this case).

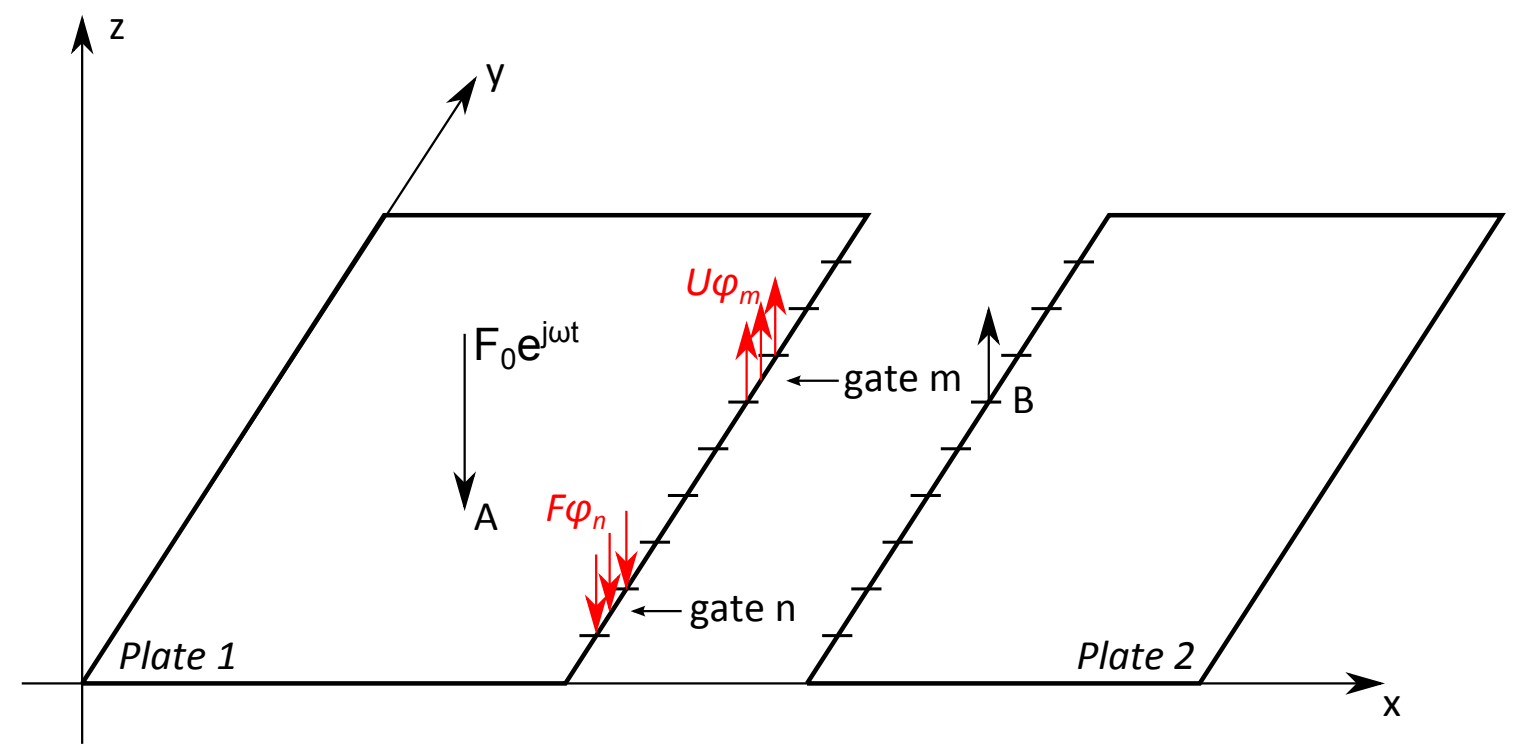

Figure 6: Two rectangular plates that are to couple along a line. Partition of the coupling line into 10 segments. 


\subsubsection{Exponential functions:}

Instead of cutting the edge into segments, the idea of projecting the displacements on exponential functions is considered:

$$
\varphi_{n}(s)=\frac{1}{\sqrt{L}} \exp \left(\frac{\jmath n \pi s}{L}\right)
$$

where $L$ is the length of the junction $\Gamma$ and $\jmath=\sqrt{-1}$. This choice is motivated by the fact that the flexural motion of a plate can be described by trigonometric functions. The associated scalar product is defined as follows, ensuring the set to be orthonormal:

$$
\langle f, g\rangle=\int_{\Gamma} f(s) g^{*}(s) d s
$$

In practice, the whole line is excited by a force that varies as a complex exponential function along its curvilinear coordinate. A term of the admittance matrix is calculated by projecting the resulting displacement over an exponential function. Setting a certain $i_{\max }$, this calculation has to be done for all the combinations $(n, m) \in \llbracket-i_{\max }, i_{\max } \rrbracket^{2}$. The free displacements are also projected on the exponential functions.

\subsubsection{Chebyshev polynomials:}

The Chebyshev polynomials are widely used to interpolate functions and are thus an interesting alternative for the condensation functions. ${ }^{22}$ They can be defined recursively, and the 5 first functions are plotted on Figure 7 with the substitution $X=\frac{2 s}{L}-1$ :

$$
\left\{\begin{aligned}
T_{0}(X) & =1 \\
T_{1}(X) & =X \\
T_{n+2}(X) & =2 X T_{n+1}(X)-T_{n}(X)
\end{aligned}\right.
$$

The scalar product associated to the Chebyshev polynomials takes into account the weight function $\frac{1}{\sqrt{s(L-s)}}$ :

$$
\langle f, g\rangle=\frac{L}{\pi} \int_{\Gamma} f(s) g^{*}(s) \frac{1}{\sqrt{s(L-s)}} d s
$$

The weight function ensures the set $\frac{T_{0}}{\sqrt{2}} \cup\left(T_{n}\right)_{n \in \mathbb{N}^{*}}$ to be orthonormal and reduces numerical errors by quadratically clustering the nodes at the end of the segment.

\subsection{Results with $N=3$ transfer functions}

The mean quadratic transverse velocity on the surface of the two plates coupled together is calculated thanks to the CTF method with the 3 different condensation functions presented previously and is compared to a reference calculation. Considering the coordinates origin 


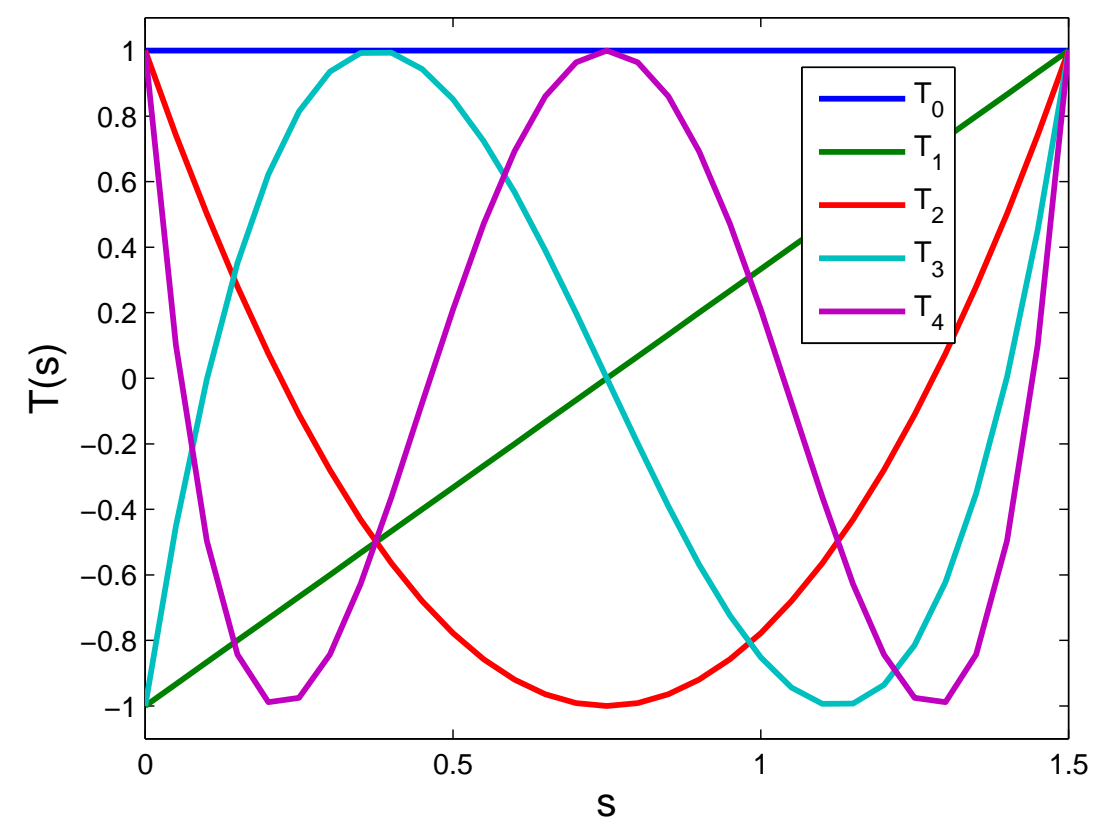

Figure 7: Chebyshev polynomials of first kind for $n \in\{0,1,2,3,4\}$. 
at the bottom left of plate 1 , the system is excited by a transverse harmonic excitation at the point of coordinates $(0.9 ; 0.5)$. On Figures 8 and 9 , the responses and the relative errors are plotted as a function of the frequency in narrow band, with 3 transfer functions for the CTF methods $(N=3)$. This criterion means:

- dividing the arc in 3 segments when the condensation functions are gates;

- taking $i_{\max }=1$ for the exponential functions, i.e.: $\varphi(s) \in\left\{\frac{1}{\sqrt{L}} \exp \left(\frac{-\jmath \pi s}{L}\right) ; \frac{1}{\sqrt{L}} ; \frac{1}{\sqrt{L}} \exp \left(\frac{\jmath \pi s}{L}\right)\right\}$;

- using the 3 Chebyshev polynomials $T_{0}, T_{1}$ and $T_{2}$;

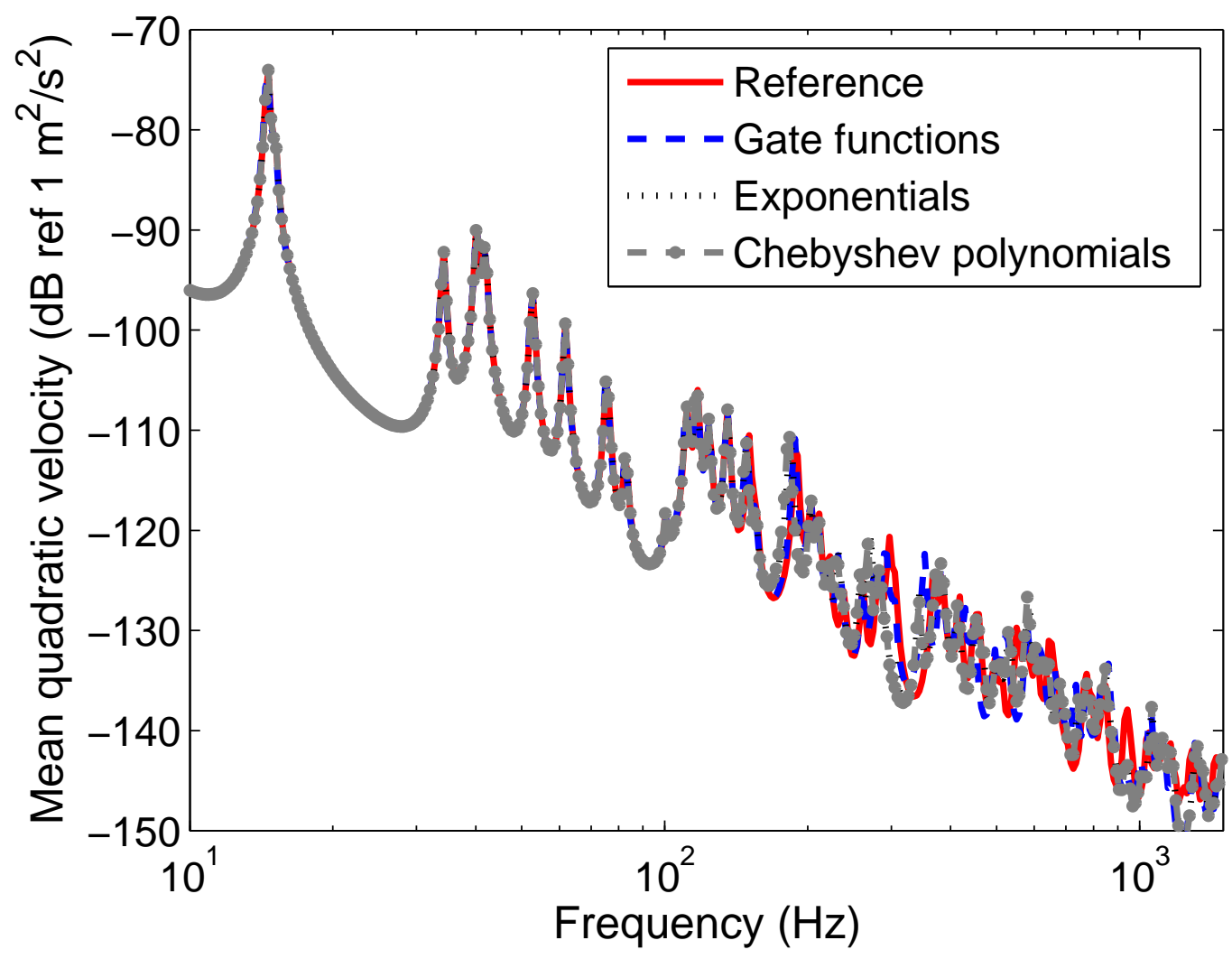

Figure 8: Mean quadratic transversal velocity of the system made of the two coupled plates and under a point harmonic load. Comparison between the reference and the CTF methods with $N=3$.

Based on the observations on Figures 8 and 9, it seems that the results fit well until around $f_{\text {lim }} \simeq 150 \mathrm{~Hz}$. For this test case, some differences up to $1 \mathrm{~dB}$ can be seen at the 


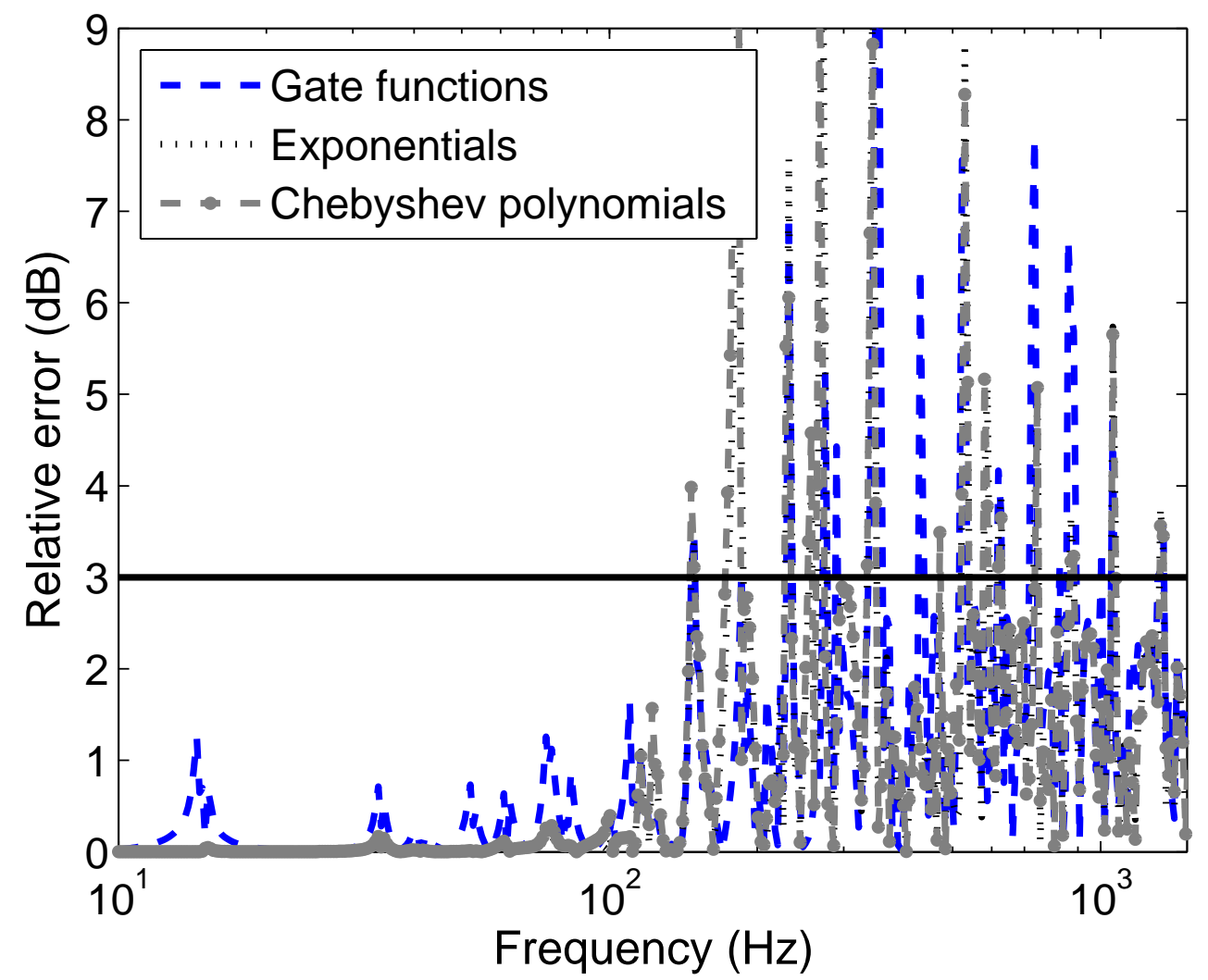

Figure 9: Relative error $(\mathrm{dB})$ of the mean quadratic transversal velocity in comparison to the FEM reference for the three CTF methods with $N=3$. 
resonance frequencies in the case of the gate functions. These differences are due to a slight frequency shift in the resonance and are acceptable for industrial applications. Above this frequency, the transfer functions are not able to describe properly the phenomena. In other terms, the forces and displacements cannot be correctly interpolated by the condensation functions at higher frequencies.

\subsection{Convergence criteria}

Assuming that at least 2 points per wavelength are necessary to sample a signal ( $c f$. Nyquist-Shannon sampling theorem), one can link the number of transfer functions $N$ with the frequency limit $f_{\text {lim }}$ ensuring convergence of the results. Considering that $\lambda_{f}$ is the flexural wavelength of the plate at $f_{\text {lim }}$, and based on the observations of the previous subsection, the convergence criteria can be written as follow:

- for gate functions, two segments are needed to describe a wavelength:

$$
L_{S} \leq \frac{\lambda_{f}}{2}
$$

- for Chebyshev polynomials, the degree of the polynomial must be at least equal to the number of points that the function needs to interpolate:

$$
N_{\max } \geq \frac{2 L_{y}}{\lambda_{f}}-1
$$

- the same reasoning can be made for exponential functions, noting that in this case $i$ can take negative values:

$$
i_{\max } \geq \frac{L_{y}}{\lambda_{f}}-\frac{1}{2}
$$

These criteria are equivalent, meaning that a frequency results in the same number of transfer functions for each condensation function. In the case of a $17 \mathrm{~mm}$ thick plate, $N=3$ leads to a frequency limit of $f_{l i m}=168 \mathrm{~Hz}$, which fits the observations on figure 8 . Applying those criteria, $N=10$ transfer functions are needed to ensure the convergence of the results above $1500 \mathrm{~Hz}$. The convergence is moreover checked on Figure 10 for the transversal displacements of points $\mathrm{A}$ and $\mathrm{B}$. The coordinates of the point $\mathrm{B}$ are $(1.2 ; 0.9)$ and the point is supposed to be located on the subsystem 2 for the CTF calculation. The plots on Figure 10 have only been presented with the CTF method using the gate functions, but the complex exponentials and the Chebyshev polynomials show good convergence too.

\subsection{Position of the excitation point}

To check whether the method is still valid when the vibration shape at the line junction becomes more intricate, the excitation is moved to the point of coordinates $(1.2 ; 0.5)$ and 

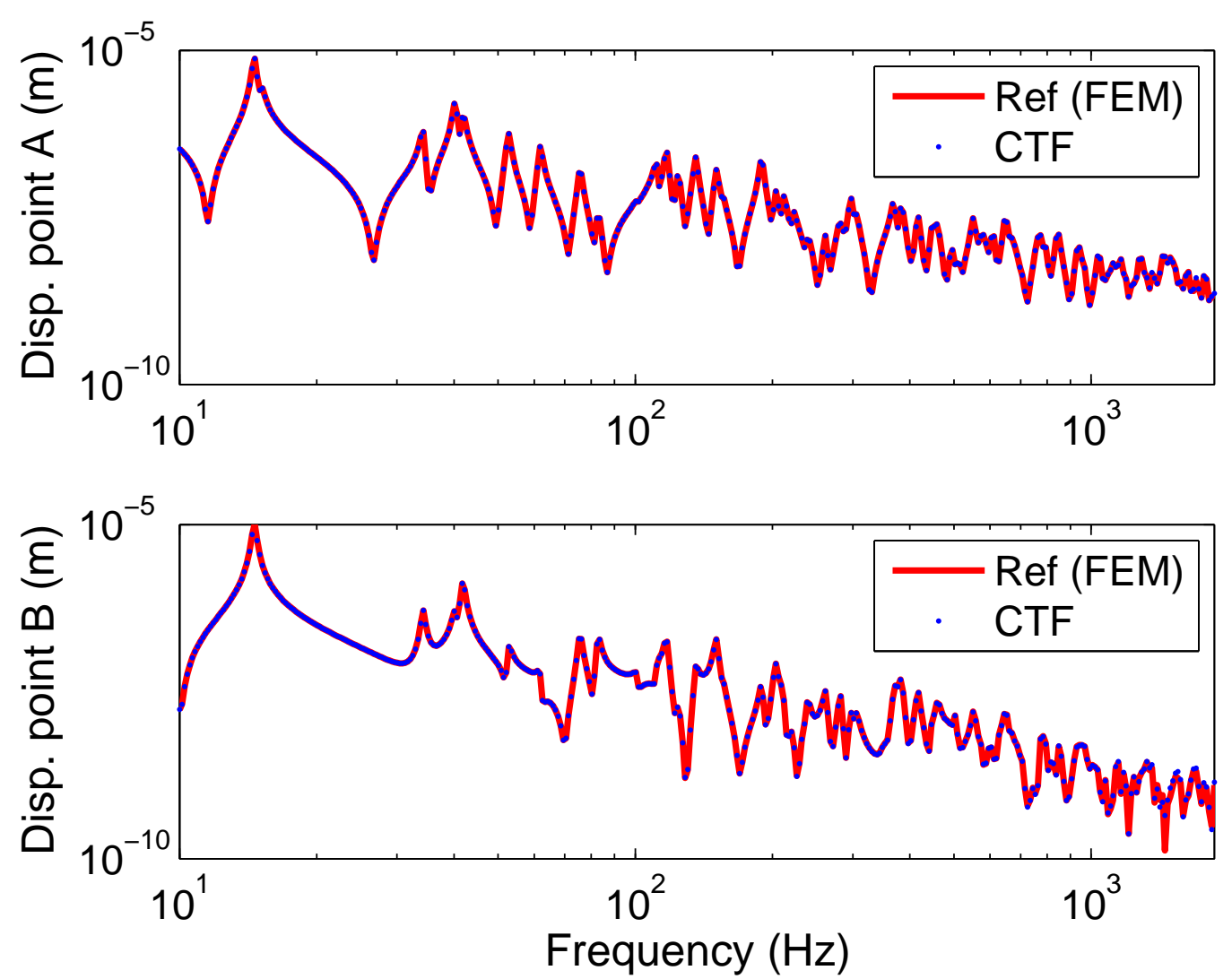

Figure 10: Transversal displacement of point A (top) and point B (bottom). Comparison between the reference and the CTF method with 10 gate functions $\left(L_{s}=0.15 \mathrm{~m}\right)$. 
lies thus on the edge of plate 1 , at the junction with plate 2 . The amplitude of the transversal displacement is plotted as a function of the abscissa on the coupling line on Figure 11, for the cases where the excitation is on or further away from the junction. The frequency of $1414 \mathrm{~Hz}$ has been chosen, because important discrepancies have been seen at this frequency for the CTF method with Chebyshev polynomials. The mesh for the finite element calculation is 5 times finer in order to have smoother curves, but the CTF calculations were still performed with the coarser mesh used previsouly in this paper. In the case where the excitation point is on the junction, the vibration shape is less regular along the line than in the case where the excitation point is further from the junction. In particular, a singularity can be seen on the red solid curve at $y=0.5 \mathrm{~m}$, corresponding to the location of the excitation point.

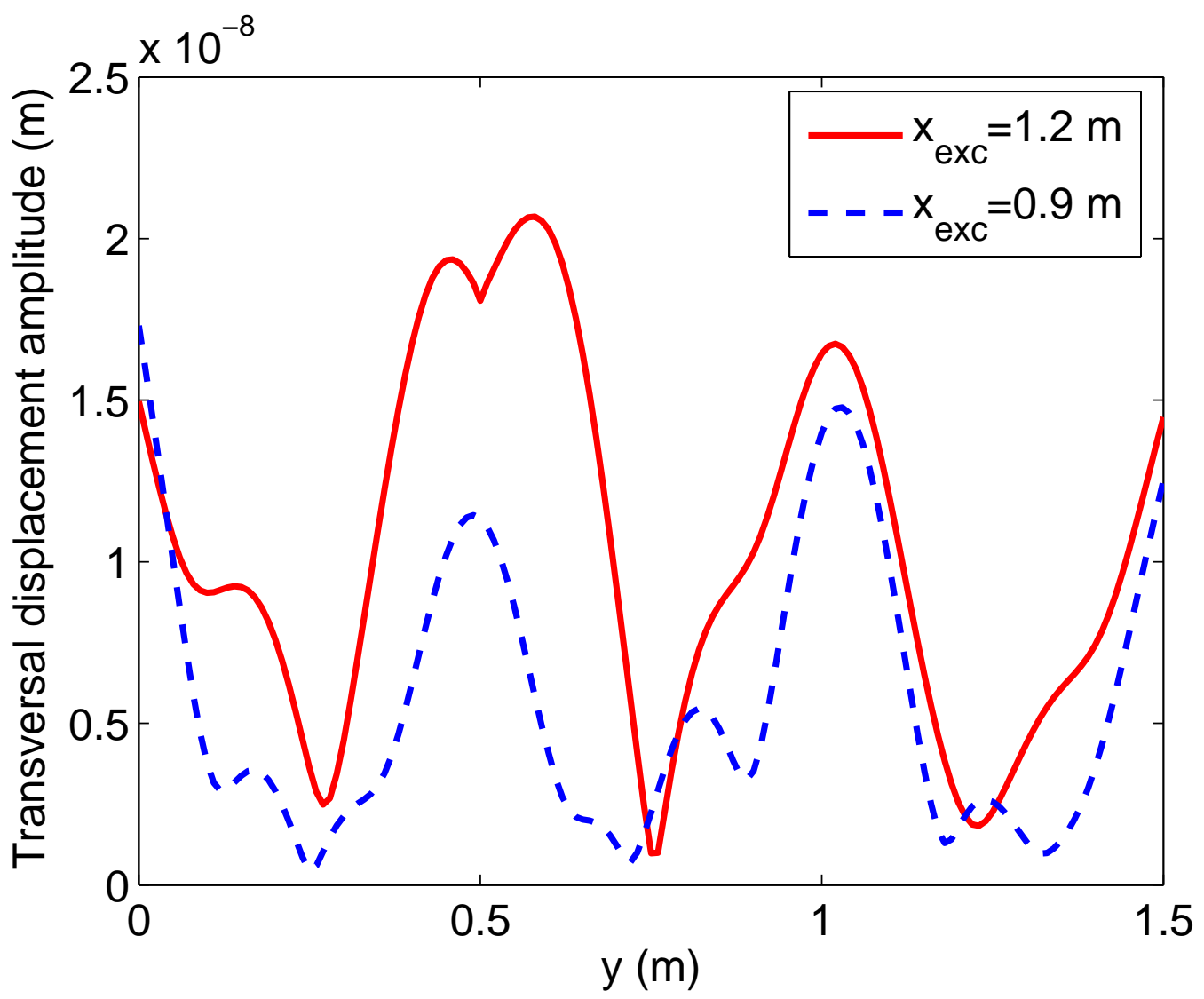

Figure 11: Amplitude of the transversal displacement along the coupling line for two different positions of the excitation point at $f=1414 \mathrm{~Hz}$.

The relative errors of the mean quadratic velocity compared to FEM reference calculations are evaluated for the two positions of the excitation point, for the three different sets 
of condensation functions. The number of condensation functions are chosen accordingly to the convergence criteria. It can be seen that the position of the excitation point has no influence on the convergence of the CTF method in the case of the gate and exponential functions (not plotted here for the sake of clarity). Nevertheless, in the case of the Chebyshev polynomials, the set is not suited to describe properly the singularity on the junction and leads to more important errors, as seen on the plot on Figure 12.

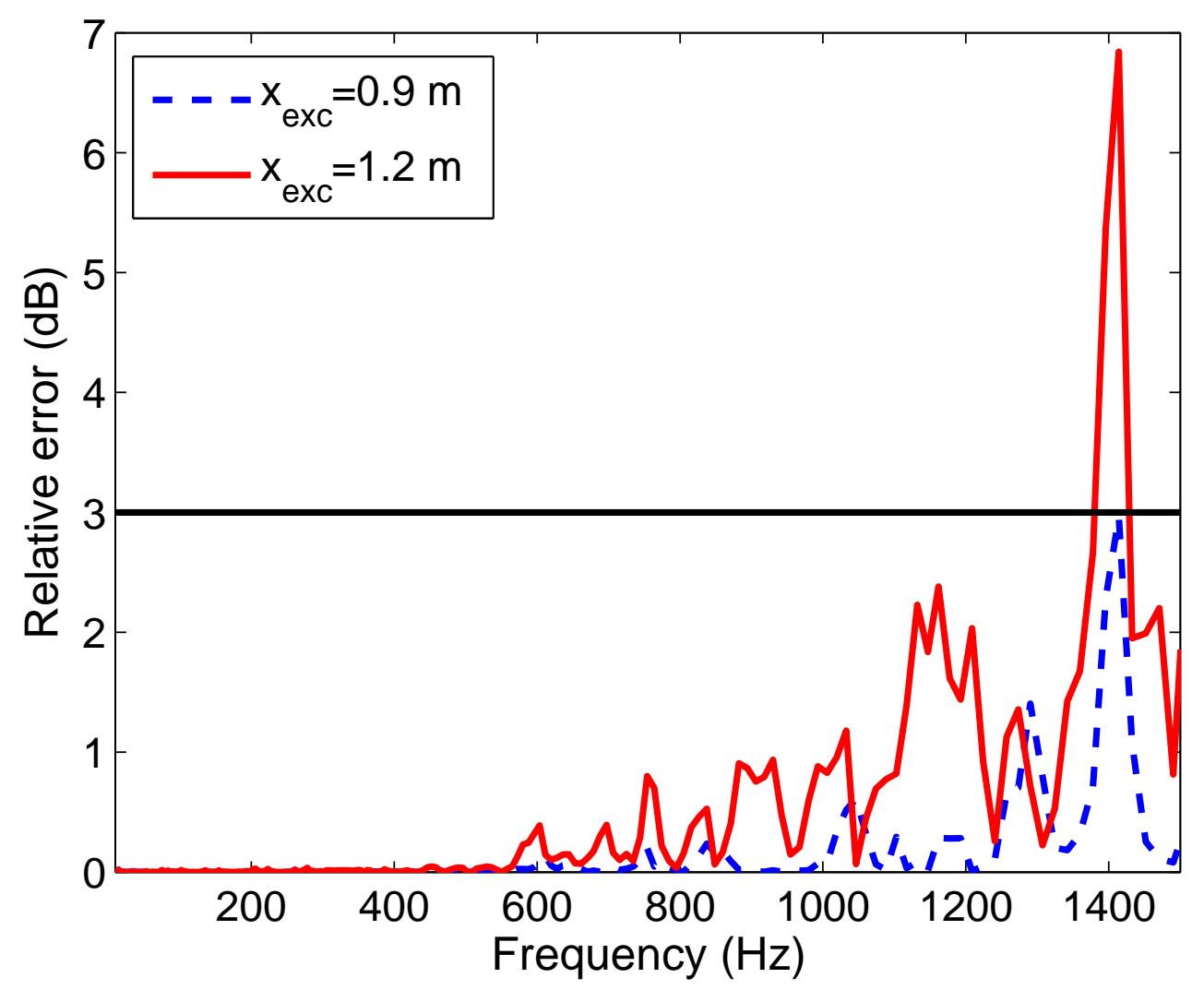

Figure 12: Relative error $(\mathrm{dB})$ of the mean quadratic transversal velocity in comparison to the FEM references for the CTF method with $N=10$ Chebyshev polynomials. Comparison for two positions of the excitation point.

As a general comment, it is thus advisable to avoid having singularities on the coupling line, to be able to approximate more easily the physical values at the junction. In other words, in the case of the Chebyshev polynomials at least, the coupling line should remain far enough from the excitation to get rid of the near-field effects. 


\subsection{Influence of the cross admittance}

One can wonder if the cross admittance terms (i.e. the extra-diagonal blocks of the admittance matrix: $n \neq m$ ) play an important role and if there is a coupling between the different condensed transfer functions. The results are plotted on Figure 13 with $N=10$ transfer functions, to ensure good convergence on the whole frequency domain. They are given for the case of full admittance matrices, and the case where the extra-diagonal blocks are set to zero. It is clear that it poorly converges in the latter case. Thus it can be said that the cross admittance is not insignificant and cannot be neglected. The results have been shown with the gate functions only but similar results are observed with other condensation functions.

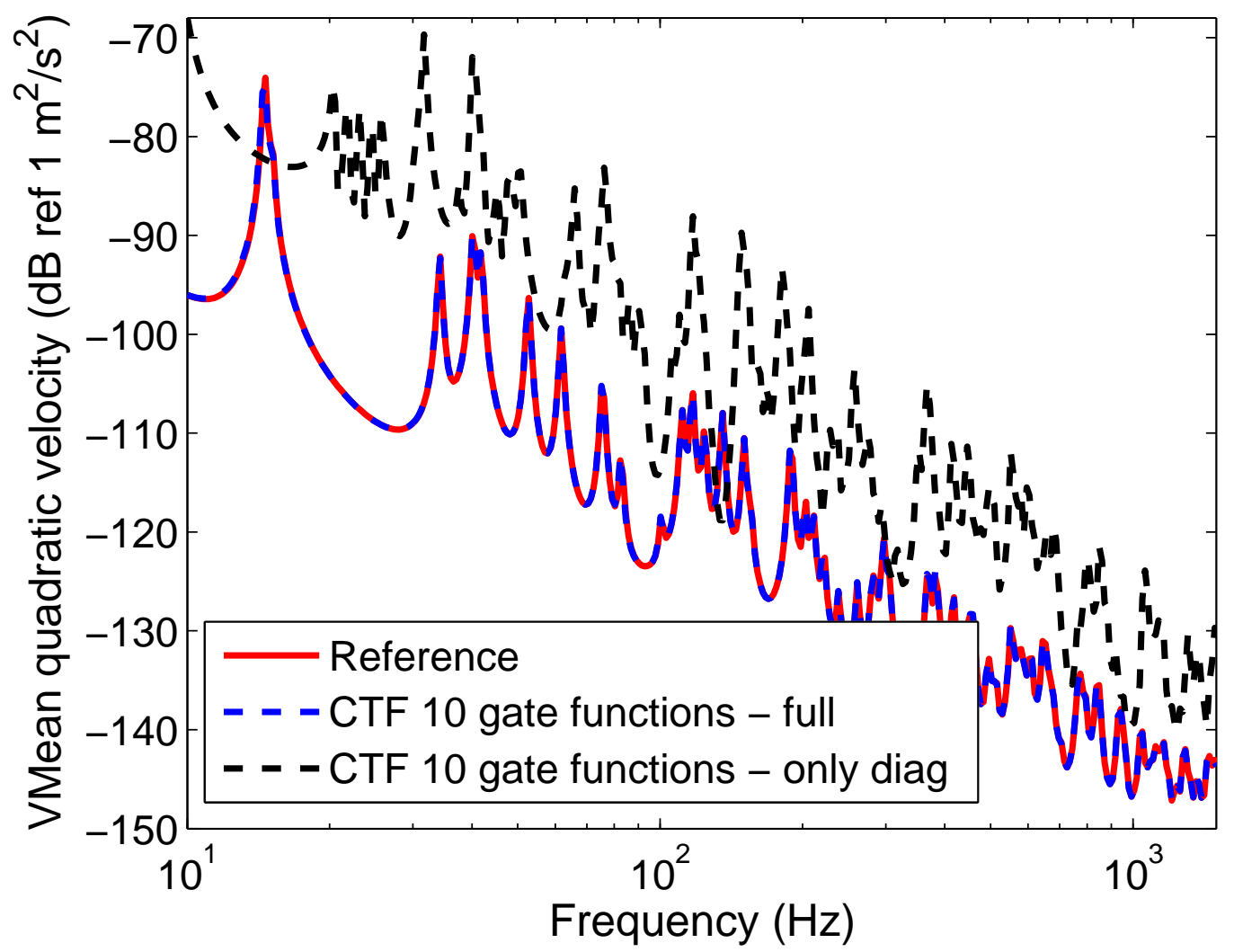

Figure 13: Mean quadratic transversal velocity on the system made of the two plates coupled and under a punctual harmonic load. 


\subsection{Numerical sensitivity}

Like most of the numerical methods, it is important to check the stability of the method with regard to the input data. As seen on Equation 10, the CTF method involves the inversion of the sum of the admittance matrices. The condition number with respect to inversion is used to check if a problem is ill-conditionned and is usually defined as the ratio of the largest singular value of the matrix to the smallest. ${ }^{23}$ If this number is particularly high, it indicates that the problem is sensitive to numerical errors.

In the case of the CTF methods with 10 transfer functions, the condition number is plotted as a function of the frequency on Figure 14. Although small fluctuations on the condition number at 734.6 and $1019 \mathrm{~Hz}$ can be seen, it can be considered that it remains around $10^{8}$ for the gate functions and the Chebyshev polynomials on the whole frequency domain . The condition number for the exponential functions is much higher (around 10 ${ }^{14}$ ) and it can thus be said that the method is more sensitive to numerical errors in this case, even if the results show good agreement with the reference calculation.

\subsection{Validation on a curved shell}

To show that the convergence criteria defined earlier in this paper remains valid in the case of subsystems coupled along non-straight line connection, a curved shell as shown on Figure [15] is considered. It has the same dimensions and material parameters as the plate presented in the beginning of the section, but a $2.5 \mathrm{~m}$ curvature is added widthwise. The excitation is normal to the shell at the point of coordinates $(0.9 ; 0.5)$, the coupling line is selected at $x=1.2 \mathrm{~m}$ and the normal mean quadratic velocity on the surface of the whole plate is calculated by FEM (reference) and by the CTF method. For the sake of simplicity and because it has proven to give the best results in the previous sections, only the set of gate functions is considered for this case.

On Figure 16, the response of the plate is recalled (black dotted line) and is compared to the response of the curved shell (red solid line). The increase in the natural frequencies of the system shows that the curvature increases the stiffness of the structure. Thus, at a given frequency, the flexural wavelength is longer in the curved shell than in the planar structure, and could result in a less restrictive criteria (i.e. wider gate functions for instance). Nevertheless, as the flexural wavelength in a curved shell cannot be calculated easily, the convergence criterion is calculated from the flexural wavelength in the planar structure of same material properties and thickness. $L_{s}=0.15 \mathrm{~m}$ wide gate functions are accordingly considered to ensure the convergence of the method. Through this example, the plot on Figure 16 shows that the CTF method is still valid on more complex cases. 


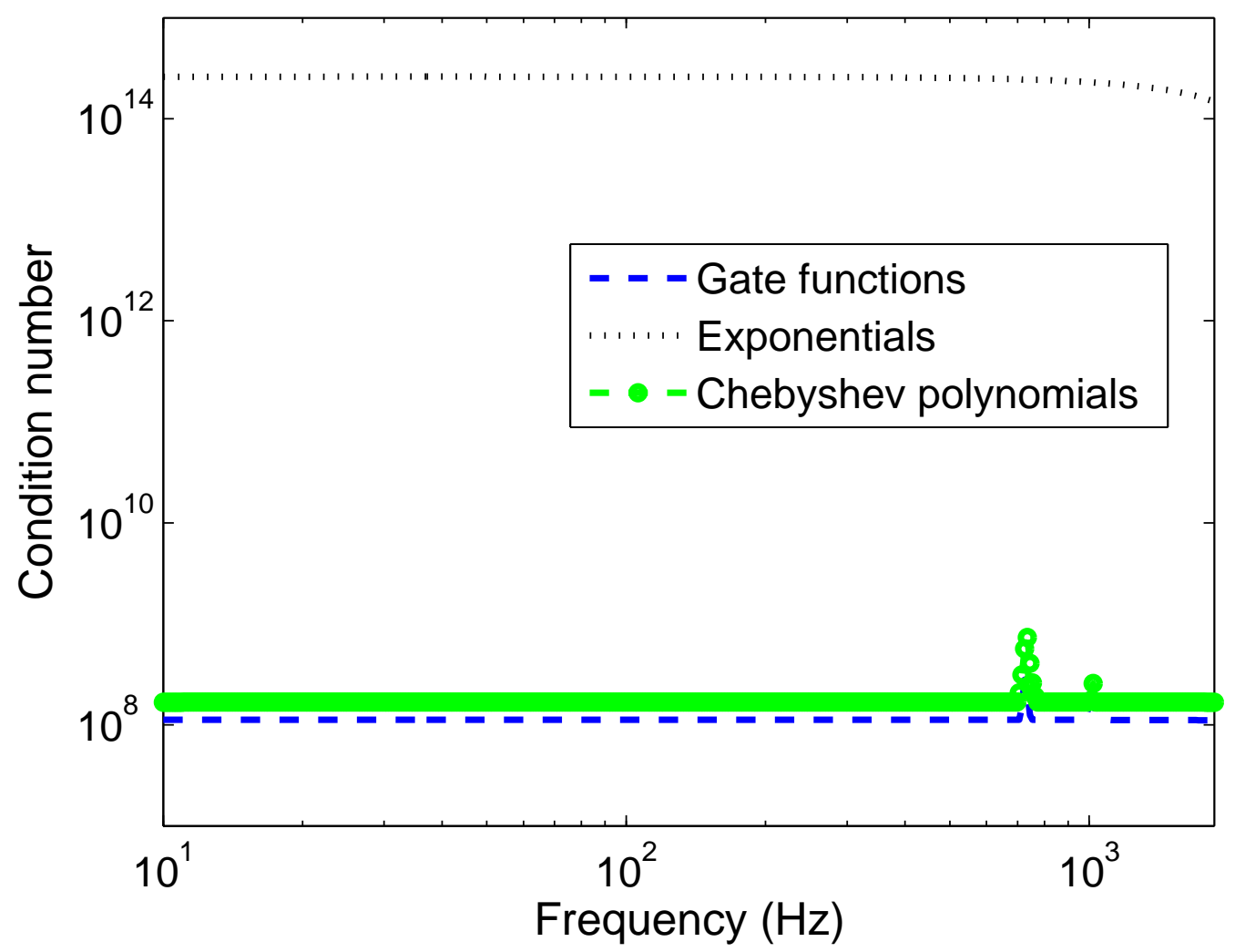

Figure 14: Condition number with respect to inversion of the sum of the admittance matrices, as a function of the frequency. 


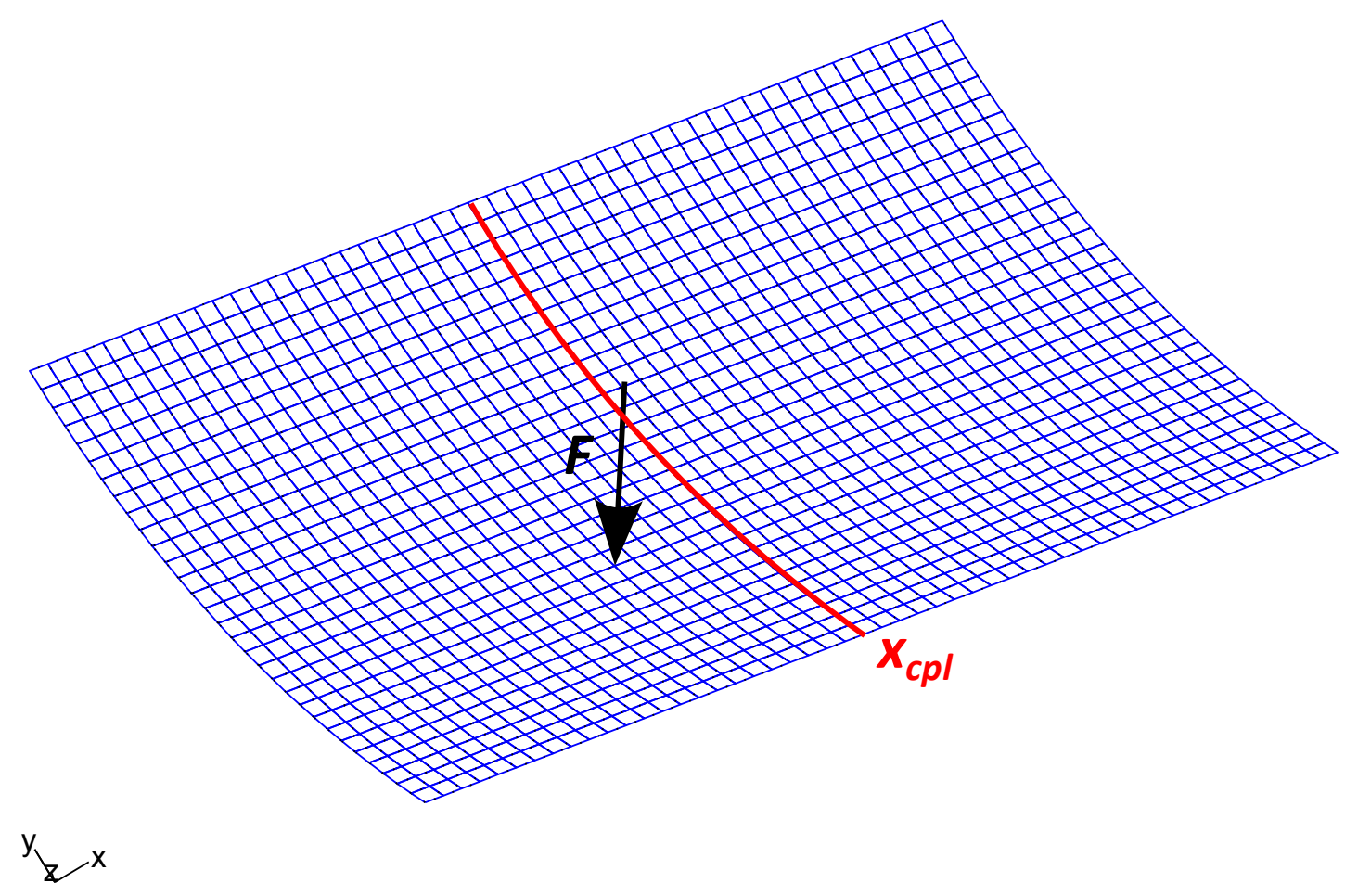

Figure 15: Mesh of a curved shell. 


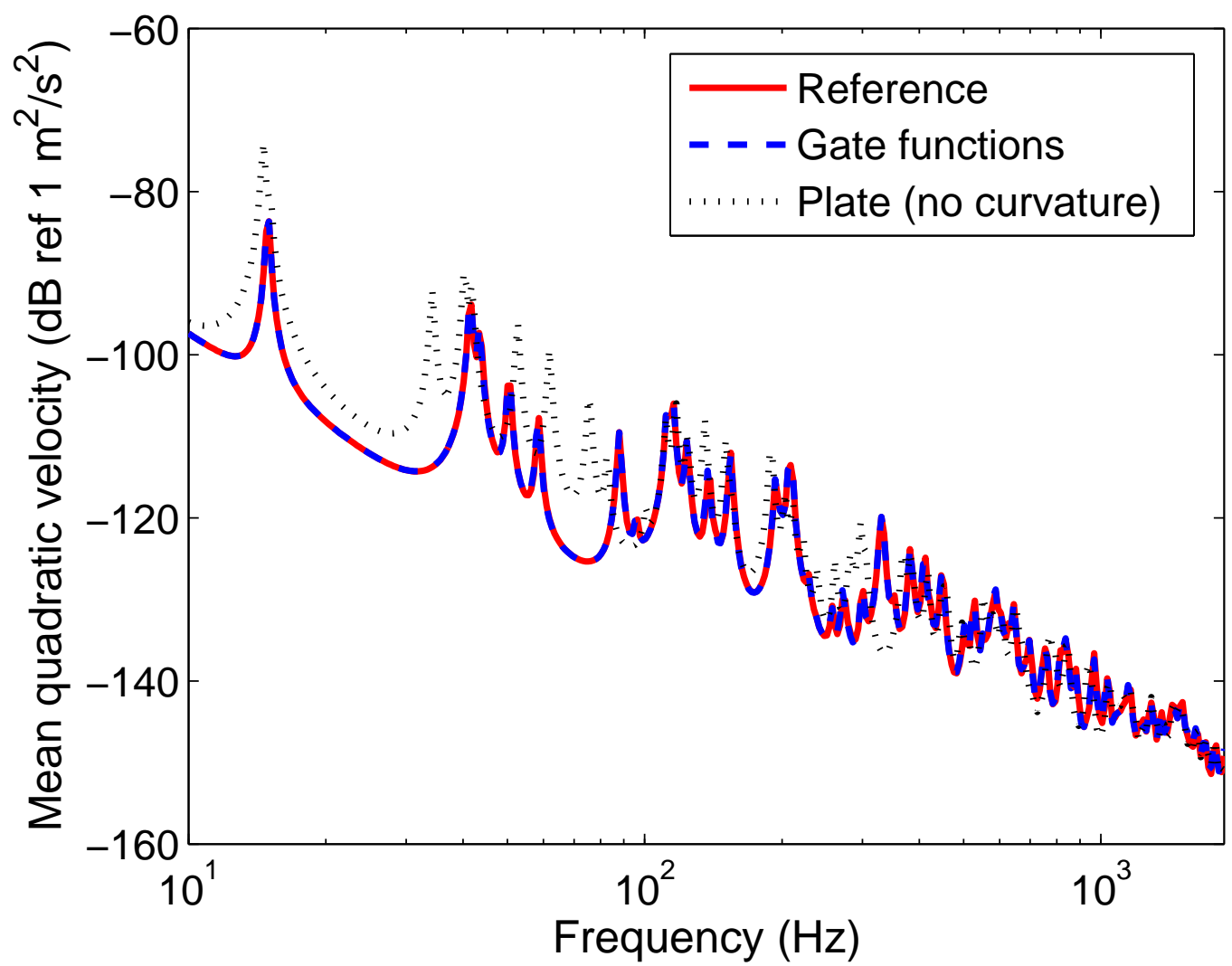

Figure 16: Mean normal quadratic transversal velocity on the system made of two curved shells and under a point harmonic load. Comparison between the reference and the CTF method with $N=10$ gate functions. 


\section{Conclusion and further work}

A sub-structuring approach based on the mobility method has been developed to couple mechanical systems along a line junction. Efforts and displacements are condensed thanks to a set of orthonormal functions and the coupling forces between the subsystems are deduced. The method has shown good results on simple structures and convergence criteria have been highlighted. In particular, the so-called gate functions seem to be more efficient in terms of numerical sensitivity and at the same time more robust to singularities. These condensation functions can be compared to the patches used in the PTF method ${ }^{10}$ in the case of the coupling with acoustic domains. Unlike classical reduction methods, such as Component Mode Synthesis ${ }^{2}$ or branch mode analysis, $\frac{3}{3}$ the subsystems in the CTF method do not necessarily need to be described by Finite Element Method (FEM) or by projections on a modal basis. Indeed, it is sufficient to be able to evaluate the admittance at the interfaces by any mean to apply the method.

The CTF method can now be further applied to the case of non-straight line connections. More particularly, it presents a great interest to add non-axisymmetric internal frames to submerged cylindrical shells: structures such as longitudinal floors or curved plates linking adjacent stiffeners can be found in industrial applications. Many studies have focused on the vibroacoustic behavior of shells immersed in water so far, but only a very few of them have considered non-axisymmetry. To tackle this issue, the idea is to couple together through CTF:

- an axisymmetric stiffened submerged shell described by the dedicated Circumferential Admittance Approach (CAA)! $\underline{24}$

- non-axisymmetric internal frames modeled by FEM to offer a great flexibility on their design.

Results showing good agreement for a test case will soon be released and the influence of the non-axisymmetric internal frames will be discussed.

\section{Acknowledgment}

This work was funded by DCNS and performed within the framework of the LabEx CeLyA of Université de Lyon, operated by the French National Research Agency (ANR-10-LABX0060/ANR-11-IDEX-0007).

\section{References}

${ }^{1}$ W. C. Hurty. Dynamic analysis of structural systems using component modes. AIAA journal, 3(4):678-685, 1965. 
${ }^{2}$ M. C. C. Bampton and R. R. Craig Jr. Coupling of substructures for dynamic analyses. AIAA Journal, 6(7):1313-1319, 1968.

${ }^{3}$ G. M. L. Gladwell. Branch mode analysis of vibrating systems. Journal of Sound and Vibration, 1(1):41-59, 1964.

${ }^{4}$ R. H. MacNeal. A hybrid method of component mode synthesis. Computers $\&$ Structures, 1(4):581-601, 1971.

${ }^{5}$ M. A. Tournour, N. Atalla, O. Chiello, and F. Sgard. Validation, performance, convergence and application of free interface component mode synthesis. Computers $\mathbb{E}$ Structures, 79(20):1861-1876, 2001.

${ }^{6}$ G. J. O'Hara. Mechanical impedance and mobility concepts. The journal of the Acoustical Society of America, 41(5):1180-1184, 1967.

${ }^{7}$ E. L. Hixson. Mechanical impedance. Shock and vibration handbook, pages 10-1, 1976.

${ }^{8}$ B. Petersson and J. Plunt. On effective mobilities in the prediction of structure-borne sound transmission between a source structure and a receiving structure, part i: Theoretical background and basic experimental studies. Journal of Sound and Vibration, 82(4):517-529, 1982.

${ }^{9}$ B. Petersson and J. Plunt. On effective mobilities in the prediction of structure-borne sound transmission between a source structure and a receiving structure, part ii: Procedures for the estimation of mobilities. Journal of Sound and Vibration, 82(4):531-540, 1982.

${ }^{10}$ M. Ouisse, L. Maxit, C. Cacciolati, and J.-L. Guyader. Patch transfer functions as a tool to couple linear acoustic problems. Journal of vibration and acoustics, 127(5):458-466, 2005 .

${ }^{11}$ J.-D. Chazot and J.-L. Guyader. Prediction of transmission loss of double panels with a patch-mobility method. The Journal of the Acoustical Society of America, 121(1):267$278,2007$.

${ }^{12}$ M. Aucejo, L. Maxit, N. Totaro, and J.-L. Guyader. Convergence acceleration using the residual shape technique when solving structure-acoustic coupling with the patch transfer functions method. Computers \& Structures, 88(11):728-736, 2010.

${ }^{13}$ L. Maxit, M. Aucejo, and J.-L. Guyader. Improving the patch transfer function approach for fluid-structure modelling in heavy fluid. Journal of Vibration and Acoustics, 134(5):051011, 2012. 
${ }^{14}$ G. Veronesi, C. Albert, E. Nijman, J. Rejlek, and A. Bocquillet. Patch transfer function approach for analysis of coupled vibro-acoustic poblems involving porous materials. $S A E$ Technical Paper, 2014-01-2092, 2014.

${ }^{15}$ M. Boucher, B. Pluymers, and W. Desmet. Coupled (vibro)-acoustic problems using geometrical acoustics and patch transfer functions. ICSV22, Florence, Italy, 2015.

${ }^{16}$ V. Meyer, L. Maxit, J.-L. Guyader, T. Leissing, and C. Audoly. A condensed transfer function method as a tool for solving vibroacoustic problems. NOVEM 2015, Dubrovnik, Croatia, 2015.

${ }^{17}$ F. A. Firestone. The mobility method of computing the vibration of linear mechanical and acoustical systems: Mechanical-electrical analogies. Journal of applied Physics, 9(6):373-387, 1938.

${ }^{18}$ S. Rubin. Mechanical immittance-and transmission-matrix concepts. The Journal of the Acoustical Society of America, 41(5):1171-1179, 1967.

${ }^{19}$ F. J. Fahy and P. Gardonio. Sound and structural vibration: radiation, transmission and response. Academic press, 2007.

${ }^{20}$ R. D. Mindlin. Influence of rotary inertia and shear on flexural motions of isotropic elastic plates. $18: 31-38$.

${ }^{21} \mathrm{~S}$. Rubin. Improved component-mode representation for structural dynamic analysis. AIAA journal, 13(8):995-1006, 1975.

${ }^{22}$ B. Fornberg. A practical guide to pseudospectral methods, volume 1. Cambridge university press, 1998.

${ }^{23}$ P. A. Nelson and S.-H. Yoon. Estimation of acoustic source strength by inverse methods: Part I, conditioning of the inverse problem. Journal of sound and vibration, 233(4):639$664,2000$.

${ }^{24}$ L. Maxit and J.-M. Ginoux. Prediction of the vibro-acoustic behavior of a submerged shell non periodically stiffened by internal frames. The Journal of the Acoustical Society of America, 128(1):137-151, 2010. 